Z Epileptol 2017 $\cdot 30: 179$

DOI 10.1007/s10309-017-0141-2

Online publiziert: 6. Juli 2017

๑) Springer Medizin Verlag GmbH 2017

CrossMark

\section{Friedhelm C. Schmitt ${ }^{1}$ Adam Strzelczyk ${ }^{2}$}

' Universitätsklinik für Neurologie, Otto-von-Guericke-Universität, Magdeburg, Deutschland

${ }^{2}$ Epilepsiezentrum Frankfurt Rhein-Main, Universitätsklinikum Frankfurt, Frankfurt, Deutschland

\title{
Staffelstabübergabe an die neuen Herausgeber
}

Mehr als groß sind die Fußstapfen, die Herr Prof. Bernhard Steinhoff und Frau Prof. Heidrun Potschka als Herausgeber der Zeitschrift für Epileptologie hinterlassen. Über mehrere Jahre haben beide die Zeitschrift entscheidend geprägt und mit der Einführung der Themenhefte die Weichen gestellt, um Heft für Heft eine klinisch und wissenschaftlich interessante Zusammenfassung für den Leser zu erstellen. Damit konnte sich die Zeitschrift als wichtige Informationsquelle für den epileptologisch interessierten Leser im deutschsprachigen Raum etablieren. Ein entscheidender Schritt in Richtung der Anerkennung als deutschsprachiges, wissenschaftlich relevantes Journal gelang beiden Herausgebern, indem die Zeitschrift für Epileptologie nicht nur in „Excerpta Medica Database" (EMBASE) und Scopus, sondern auch in den „Emerging Sources Citation Index" (ESCI) aufgenommen wurde. Der Staffelstab zur Herausgeberschaft wurde vorausschauend und wohlgeordnet bei der diesjährigen Mitgliederversammlung der Deutschen Gesellschaft für Epileptologie e.V. (DGfE) während der Dreiländertagung in Wien übergeben.

Dort wurden als neue Herausgeber wir, Dr. Friedhelm C. Schmitt aus Magdeburg und Prof. Adam Strzelczyk aus Frankfurt am Main, gewählt. Unser Ziel ist es, die erfolgreiche Arbeit unserer Vorgänger fortzuführen, und daher werden wir die Erstellung von Themenheften unverändert beibehalten. Hierfür bleiben wir auf die Mitarbeit der Gastherausgeber angewiesen, ohne die in der Vergangenheit und in der Zukunft die Erstellung der Themenhefte nicht möglich gewesen wäre bzw. sein wird. Ideen und Anregungen zur Gestal- tung der nächsten Hefte sind herzlich willkommen, und wir freuen uns auf Ihre Zuschriften. Das Gleiche gilt für Leserbriefe zu publizierten Beiträgen, die mehr als willkommen sind, da diese den lebhaften Austausch in der Epileptologenszene ermöglichen und zur besseren Einordnung der Beiträge in den wissenschaftlichen oder klinischen Kontext verhelfen. Die regelmäßigen Beiträge in der altbekannten Rubrik der „Der interessante Fall“ soll weiter unter der Section-Editorenschaft von Prof. Bernhard Steinhoff erscheinen; für die Rubrik „Der epilepsiechirurgische Fall“ konnten wir als neue „section editors“ Frau Prof. Susanne Knake aus Marburg und Frau PD Georgia Ramantani aus Zürich gewinnen. Beide freuen sich über eingereichte Beiträge und werden in den nächsten Wochen epilepsiechirurgisch tätige Kolleginnen und Kollegen um Vorschläge bitten. Zudem möchten wir ein Format einführen, das es - ähnlich, wie z. B. „Clinical Picture“ in Lancet - ermöglichen soll, mit einer sehr begrenzten Zahl an Wörtern und Referenzen eine interessante Befundkonstellation, entweder bildmorphologisch, elektrophysiologisch und videometrisch, $\mathrm{zu}$ veröffentlichen. Das entsprechende Format wird in Kürze bei den „Hinweisen für Autoren“ auf der Internetseite http://www.springer.com/medicine/ neurology/journal/10309 nachzulesen sein.

Im Namen des Vorstands der DGfE danken wir Herrn Prof. Bernhard Steinhoff und Frau Prof. Heidrun Potschka ausdrücklich und herzlich für ihre Tätigkeit. Beide wollen uns als Ratgeber erhalten bleiben und werden auch im
Beirat der Zeitschrift an ihrer weiteren Entwicklung beteiligt sein.

Unser gemeinsamer Dank gilt nicht zuletzt dem Springer Medizin Verlag und seinen Mitarbeitern, insbesondere Frau Petra Elster, für die professionelle, unkomplizierte Zusammenarbeit während des Wechsels der Herausgeberschaft.

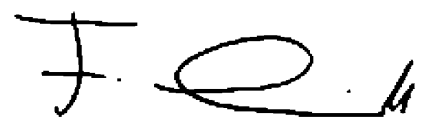

Friedhelm C. Schmitt, Magdeburg

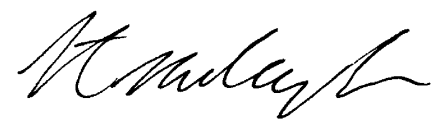

Adam Strzelczyk, Frankfurt am Main

\section{Korrespondenzadresse}

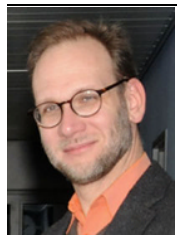

Dr. F. C. Schmitt

Universitätsklinik für

Neurologie, Otto-von-

Guericke-Universität

Leipziger Str. 44,

39120 Magdeburg,

Deutschland

fc.schmitt@med.ovgu.de

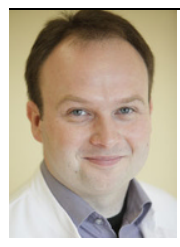

Prof. Dr. A. Strzelczyk, MHBA

Epilepsiezentrum Frankfurt Rhein-Main,

Universitätsklinikum

Frankfurt

Schleusenweg 2-16, 60528 Frankfurt, Deutschland strzelczyk@ med.uni-frankfurt.de

Interessenkonflikt. F.C. Schmitt und A. Strzelczyk geben an, dass kein Interessenkonflikt besteht. 